Public Abstract

First Name:Jun

Middle Name:

Last Name:Tao

Adviser's First Name:Chengshan

Adviser's Last Name:Xiao

Co-Adviser's First Name:

Co-Adviser's Last Name:

Graduation Term:SS 2010

Department:Electrical Engineering

Degree:PhD

\title{
Title:ROBUST RECEIVER DESIGN FOR RF COMMUNICATION AND UNDERWATER ACOUSTIC COMMUNICATION
}

This dissertation includes my work on wireless communication with the Department of Electrical and Computer Engineering at the University of Missouri, from August 2006 to July 2010. It consists of two main parts addressing robust receiver design for two different types of wireless communications: wireless radio frequency (RF) communication and wireless underwater acoustic (UWA) communication. Compared with more traditional wireless RF communication, wireless UWA communication is much more challenging, where acoustic waves instead of electromagnetic waves is used for transmitting signal in the water.

In the first part, four different topics relating to receiver design are investigated for future wireless $\mathrm{RF}$ communication using orthogonal frequency-division multiplexing (OFDM) technology and multiple-input, multiple-output (MIMO) technology. It starts with a fundamental problem on channel modeling, which is the basis for studying the other three topics. Based on the proper channel modeling, a practical Doppler spread estimation method is proposed for mobile OFDM systems. The last two topics deal with channel estimation for OFDM systems, and turbo equalization for MIMO systems. Theoretical analysis and numerical simulation have been provided throughout this part.

In the second part, two receiver schemes are proposed for high data rate UWA communication. The first scheme is a combination of conventional equalization method with a novel phase-compensation algorithm. It handles undesirable phase rotations in the received acoustic signal. The second scheme has more powerful detection capability by using iterative equalization technology. Performance evaluation on the proposed receiver schemes relies on experimental data. In this dissertation, experimental results for four different undersea trials have been reported, which demonstrates the robustness and high performance of the proposed receiver designs. 